\title{
LA RÉSILIENCE : Émergence et Conceptualisation du Phénomène
}

\section{Radja Bouzeriba}

\section{Radja Bouzeriba, Université de Skikda, Algerie.}

\section{Résumé :}

Dans cet article, nous tentons de retracer l'émergence ainsi que l'évolution de la « Résilience »,concept récemment adopté par la psychologie et désignant la capacité de reprendre son développement en dépit de traumatismes psychiques. L'individu puise ainsi dans ses ressources personnelles et environnementales pour pouvoir dépasser l'adversité. La discussion des différentes approches théoriques tourne autour de la conceptualisation de ce phénomène et de la description des différents aspects intervenant dans son processus dynamique. N'étant donc pas statique, la résilience serait une résultante d'un équilibre issu de l'interaction entre les facteurs de risques et les facteurs de protection.

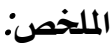

حاولنا من خلال هذا المقال، تقديم عرض بسيط حول نشأة و تطور "الرجوعية. هذا

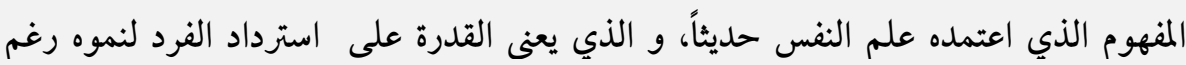

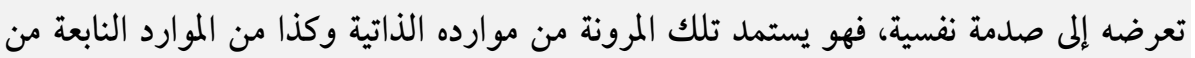
محيطه. وتدور المناقشة النظرية في هذا المقال حول يختلف المقاربات المفاهيمية التى تناولت هذه الظاهرة و حول تقديم وصفاً لمختلف الأوجه المتدخلة في سيرورتها الدينامية. و كونها

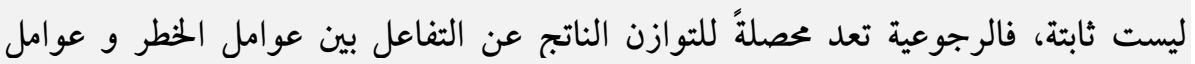
الحماية لدى الفرد. 


\section{Introduction:}

L'adoption du nouveau concept de « résilience » en psychologie a été puisée dans d'autres disciplines. Et s'il désigne à présent un phénomène psychologique évoquant la reprise du cours normal de la vie après un traumatisme psychique, il a pu signifier plusieurs autres phénomènes dans d'autres domaines. A ce titre nous prenons l'exemple de l'agronomie, où elle décrit la fertilité d'un terrain incendié, ou encore en informatique où elle définit la capacité d'un système à fonctionner malgré la présence d'anomalies dans l'un ou l'autre des éléments constitutifs. (B. Cyrulnik, 2011, p. XI)

C'est à partir des années 1980 qu'il y a eu une importante prépondérance des publications liées à ce concept, et cela pas uniquement en psychologie, mais en sociologie, en biologie, en linguistique et en anthropologie.

L'idée nouvelle qu'apporte la résilience est qu'un sujet traumatisé ne soit pas consigné à passer le reste de sa vie avec son traumatisme et les blessures profondes qui en découlent. Mais qu'il pourrait s'en sortir et dépasser sa douleur et son malheur quel que soit l'ampleur de l'évènement traumatique, et s'inscrire dans le processus de résilience.

Le phénomène de résilience décrirait en fait, la retranscription d'un destin moins sombre et un peu plus heureux, voire même réussi, où la personne évoluerait avec de nouveaux horizons, optimisant une vie nouvelle. Ce changement de trajectoire optimiste ne signifie pas la 
négligence de la souffrance des personnes et encore moins l'ignorance de leurs blessures. Les individus résilients conservent des traces de leur trauma mais ils tentent de continuer à poursuivre un autre type de développement.

Être résilient, n'est donc pas être invulnérable, ce modèle, comme l'a décrit Marie Anaut(2008, p. 12)se situe au carrefour de nombreuses et différentes variables.

Il s'avère difficile de préciser les contours théoriques de ce concept, mais nous tenterons à travers cet article de donner un aperçu sur les origines anglo-saxonnes et francophones de la résilience, avant de rapporter les plus importantes conceptions théoriques autour de la résilience, pour ensuite en décrire le contexte du profil résilient. Et enfin, nous allons discuter la dynamique résiliente au regard d'un équilibre entre les facteurs de risques et les facteurs de protection.

\section{Les origines de l'approche de la résilience :}

L'approche de la résilience est née au carrefour de différentes théories, développementales, comportementales, psychanalytiques, psychopathologiques et socioéducative. Anaut (2006, p.81), précise dans cette perspective, que « les questions relatives à la résilience rencontrent. un vif intérêt en psychologie et psychopathologie, mais également auprès de disciplines connexes (éthologie, sociologie, sciences de l'éducation, médecine.. ». C'est dire que la résilience trouve son origine dans le croisement de tous ces fondements théoriques. 
Ce concept signifie qu'il désigne la reprise du cours de la vie lorsque celle-ci a été frappée par un événement traumatique, se réfère à un processus complexe qui résulte de l'interaction des individus avec leur environnement. Ce processus ne pouvant se déclencher qu'en présence d'un agent traumatogène.

Nous ne pouvons donc parler de résilience que s'il y a eu traumatisme. Ceci peut concerner l'individu comme il peut concerner le groupe social ou familial. Un sujet traumatisé pourrait être donc résilient s'il puise dans ses ressources intrinsèques et extrinsèque pour dépasser des évènements traumatisants survenus au cours de sa vie et ayant pu tolérer, sans grands dommages des effets adverses. (Anaut, 2008, p. 34).

Le terme de résilience est issu du latin « resilientia », son origine revient à la physique où il est employé pour désigner la résistance des matériaux aux chocs violents. D’après le dictionnaire petit Larousse la résilience représente : « la caractéristique mécanique qui définit la résistance aux chocs d'un matériau». (Le petit Larousse illustré, 2008, p. 882).

A l'origine le concept de résilience désignait donc, l'élasticité et la fragilité de ces matériaux après avoir subi un choc ou une pression continue. Mais plus tard il a été emprunté à la physique et employé en psychologie comme le définie Norbert Sillamy (2006, p.228). "La capacité de vivre et de se développer en dépit de circonstances difficiles; défavorables, voire désastreuses ». L. Bailly (2006, p. 223), a pu également dans «Résilience et 
Psychanalyse » décrire cette élasticité comme une capacité à sauter en arrière dans le sens de reprendre sa place initiale et la qualifié de «l'acte de rebondir », c'est-à-dire de reprendre sa forme ou sa forme originale.

Cette résilience considérée comme capacité ou processus, n'est pas constante ni définitive, car un sujet peut être résilient pendant une période donnée et dans certains cas bien précis. Cette instabilité pourrait être due aux facteurs du moment que cela soit sur le plan individuel, familial ou extrafamilial.

Toutefois, beaucoup de chercheurs (Manciaux et al, 2001) considèrent la résilience comme un potentiel présent chez chaque individu et qu'il se développe en fonction de son évolution psychologique et des circonstances de sa vie. L'individu serait doté de ressources qui pourraient être stimulées par lui-même, ou par d'autres intervenants dans son environnement familial, scolaire, professionnel ou social.

\section{a) L'origine anglo-saxonne de la résilience :}

L'abord du concept de résilience a été effectué par différents chercheurs, mais il a débuté par les travaux anglo-saxons et nordaméricains bien avant son abord par les chercheurs français. Rutter, Werner ou encore Garmezy, ont précédé les chercheurs français tel que Cyrulnik et Manciaux et bien d'autres chercheurs dans l'étude de ce nouveau concept de résilience. 
Toutefois l'origine de l'émergence de la résilience est plus ancienne, elle remonte aux premiers travaux sur l'attachement. Bowlby aurait utilisé ce terme de résilience pour désigner « le ressort moral » (d'après une référence faite par Manciaux et col), qui signifie « la qualité d'une personne qui ne se décourage pas, ne se laisse pas abattre ». (M. Anaut, 2008, p.37).

Bowlby a évoqué la possibilité chez les individus de combattre leur mal en prenant du courage et on ne baissant pas les bras. Cette capacité morale à prendre un nouvel élan dans la vie, donne à la personne une vision positive de son évolution dans l'avenir et ainsi la force de continuer en dépit de toutes adversités, et c'est cela même le sens de la résilience.

Les anglo-saxons ont donc été les précurseurs dans le domaine de la résilience. Rutter et Garmezy ont posé les bases théoriques et les principes méthodologiques des recherches dans ce domaine, et leur ouvrage « Stress coping and développement » en est témoin, parmi d'autres recherches effectuées par la suite sur la résilience chez l'enfant et l'adolescent.

D'autres chercheurs américains ont contribué à l'émergence de l'approche de la résilience. Les travaux d'Emmy Werner, par exemple, ont beaucoup apporté à l'étude de la résilience. Elle a observé un groupe d'enfant vivant dans des conditions socioaffectives défavorables (pauvreté, violence, discorde, psychopathologie parentale, etc.), depuis la naissance jusqu'à l'âge adulte.Elle est arrivée à constater qu'un certain nombre de ces enfants, 
dits à risque, ont pu s'adapter à leur environnement social et vaincre les conditions d'adversité dans lesquelles ils vivaient. Et malgré que cette étude ne rentrent pas parmi les recherches engagées dans l'étude de la résilience, mais les résultats obtenues au terme de ses travaux chez le groupe d'enfants étudié, indiquerait qu'ils ont un fonctionnement type d'un individu résilient. Cette étude longitudinale a permis également la description du processus de la résilience comme étant la capacité des individus à favoriser un équilibre évolutif grâce à différents éléments internes, puisés dans l'un des constituants de la personnalité du sujet et externes provenant de son environnement.

\section{b) L'origine francophone de la résilience :}

Le concept de résilience malgré qu'il soit né ailleurs que dans les pays de la francophonie, a pu connaitre un front succès, ceci apparait dans le nombre de publications, des séminaires et des colloques consacrés à ce sujet.

Au début de son émergence (les années 1980),ce concept a été approché comme étant le contraire du concept de «vulnérabilité », qui lui était déjà connu en psychologie. Tomkiewicz et Cyrulnik lui ont consacré tout un chapitre dans leur ouvrage "l'enfant et sa santé » (1987) sans mentionner le terme de "résilience », et ceci quatre ans après qu'Emmy Werner ait parlé de ce concept.

C'est donc Boris Cyrulnik qui a également contribuéà l'étude de ce phénomène et à son expansion dans les pays francophones, et ce à travers ses multiples ouvrages et articles, qui relatent des faits et des évènements traumatisants vécus par différents sujets qui étaient 
capables de les surmonter et de reprendre le dessus ; êtres résilients. Nous citons parmi eux : «Les Vilains Petits Canards », « Ces Enfants Qui Tiennent Le Coup », « De Chair et d'Âme », «Un Merveilleux Malheur », « Le Murmure des Fantômes », etc.

Les premiers travaux ont porté sur l'affinement de la définition de ce concept et sur les caractéristiques des enfants à qui peut-on l'appliquer. Dans « la résilience : le réalisme de l'espérance », Stanislaw Tomkiewicz, a essayé de nous donner une définition de la résilience mais en démontrant qu'elle peut avoir trois tableau clinique :(S. Tomkiewicz, 2002, p-p. 53-55).

Le premier groupe de résilients concernerait les individus qui auraient pu avoir des conduites associales et dangereuses menaçant leur propre vie et la vie des autres, mais ils sortent vainqueur de cette épreuve. Le deuxième groupe concernerait tout être pouvant survivre de manière autonome et normale, qu'il devienne « socialement correct ».Le troisième groupe est celui des sujets victimes d'un grand traumatisme, un malheur profond, et qui réaliseraient des exploits remarquables, tel que bon nombre de scientifiques, écrivains notoires, ou encore d'hommes politique imminents.

\section{La Résilience : Conceptualisation et profilage}

Il faut reconnaitre la difficulté à délimiter les contours théoriques de ce concept, la résilience tend à être confondue et s'articule avec plusieurs autres concepts (invulnérabilité, coping, adaptation, etc.). 
Cependant, cette notion se précise et se clarifie au-fur-et-à-mesure des recherches, c'est le cas de n' importe quel autre concept qui parait flou à son émergence pour devenir opérant au fil du temps et de la pratique.

\section{a) L'approche conceptuelle du phénomène de la résilience :}

La résilience a été abordée différemment par les auteurs, et ceci apparait dans la variation de son approche. Certains la qualifieraient de capacité, d'autres comme un mode de fonctionnement, ou encore d'un processus dynamique.Rutter l'avait définie comme étant « la capacité de réussir de manière acceptable pour la société en dépit d'un stress que comporte normalement le risque grave d'une issue négative»(M. Lemay, 2001, p. 138.).

Cyrulnik, dans son ouvrage « un merveilleux malheur »a donné une définition semblable de la résilience : « la capacité à réussir à vivre et à se développer positivement, de manière socialement acceptables, en dépit du stress ou d'une adversité qui comporte normalement le risque grave d'une issue négative » (B. Cyrulnik,1999, p.10).

Nous pouvons comprendre, à travers ces deux définitions précédentes, que la personne résiliente serait capable de continuer à vivre et à se développer positivement malgré les conséquences négatives qu'aurait pu engendrer l'affrontement à des situations adverses. Ici la notion qui revient est celle de « capacité » de réussir et de vaincre, la résilience serait donc une capacité, un pouvoir que 
détient celui qui est résilient sur une situation donnée pouvant être à l'origine de sa détresse.

Anaut, renforce cette idée en décrivant la résilience comme « la capacité de sortir vainqueur d'une épreuve qui aurait pu être traumatique avec une force renouvelée. La résilience impliquant l'adaptation face au danger, le développement normal en dépit des risque et le ressaisissement de soi après un traumatisme ». (M. Anaut, 2003, p.7).

Elle apporte également la notion de « force renouvelée », la personne aurait à puiser dans ses éléments internes pour retrouver une nouvelle puissance et de pouvoir agir sur soi et sur l'environnement traumatique pour réussir à s'adapter et à retrouver son équilibre.

Nous ajoutons la définition suivante de la résilience proposée par Manciaux : «la capacité d'une personne ou d'un groupe à se développer bien, à continuer à se projeter dans l'avenir en dépit d'évènement déstabilisant, de conditions de vie difficiles, de traumatismes parfois sévères »).

Dans cette définition Manciaux a également considéré la résilience comme étant la capacité à avoir un développement normal malgré les conditions défavorables, le traumatisme et les évènements déstabilisants. Mais il apporte le fait qu'un individu résilient se « projette dans l'avenir », il aurait donc une vision optimiste ; non seulement il arrive à dépasser le passé douloureux, à vivre le présent difficile, mais il réussirait à se reconstruire un avenir. 
Ces définitions citées plus haut, démontrent bien la capacité des individus résilients à se construire, à continuer leur développement positivement et à affronter les évènements défavorables et déstabilisants pouvant entraver leur adaptation dans la société. La résilience serait une capacité donnant au sujet des caractéristiques lui permettant de produire des réponses adéquates en présence d'agents traumatogènes.

Si l'on se tenait à cette approche, l'individu aurait ou pas, la capacité de résilience, cela pourrait également sous-entendre un caractère définitif d'acquisition de la capacité de résilience. Hors-que nous savons que la résilience est variable dans le temps et selon l'évènement, en plus d'autres facteurs intrinsèques et extrinsèques, de risques et de protection, que nous développerons un peu plus bas.

D'autres chercheurs la verraient plutôt comme un résultat d'un fonctionnement. Un sujet aurait donc un fonctionnement résilient s'il manifeste un ensemble de comportements réputés par leur efficacité dans le domaine du bon développement et l'affrontement de situations traumatisantes. Dans cet abord Mastern a écrit : « la résilience réfère à une classe de phénomènes caractérisés par de bons résultats en dépit de menaces sérieuses pour l'adaptation ou le développement », selon Anaut (2008), cette définition supposerait l'existence d'une sorte de boite noire qui conduirait à ce résultat comportemental.

L'acquisition $\mathrm{du}$ fonctionnement résilient supposerait donc l'acquisition d'une structure statique et la réduirait à un caractère figé. Ceci s'oppose à l'idée que la résilience nait de l'interaction de 
plusieurs aspects individuels et environnementaux, et qui sont en perpétuel changement et progression. C'est cela, que nous proposent d'autres chercheurs et qui mettent en évidence l'interaction qu'il y a entre les facteurs internes et externes et leurs rôles dans l'apparition du phénomène de résilience.

Vanistendael, écrit dans son ouvrage « Le bonheur est toujours possible, construire la résilience », que : « la résilience n'est pas une caractéristique de l'individu au sens strict du terme, mais de la personne en interaction avec son environnement humain. Nous pouvons concevoir la résilience à partir de l'individu, puis en cercle concentrique toujours plus vaste, jusqu'à l'ensemble de la société » (A. THEIS, 2006, p. 35).

Vanistendael, considère la résilience comme une capacité qui serait la résultante d'interaction entre l'individu et son environnement, conférant à l'individu la possibilité de se saisir de son monde et d'interagir avec lui dans différentes situations. De ce fait, la résilience se constituerait de l'interaction des différents facteurs individuels, ceux émanant de son environnement proche puis s'étends vers la société dans laquelle il évolue.

Ajoutant ce que Manciaux et ses collaborateurs, ont avancés à cet abord : «A chaque instant la résilience résulte de l'interaction entre l'individu lui-même et son entourage, entre les empreintes de son vécu antérieur et le contexte du moment en matière politique, économique, sociale, humaine ».(A. THEIS, 2006). 
Pour ainsi renforcer l'idée que la résilience ne s'opère pas sur le plan individuel strict, et que le contexte dans lequel vit le sujet est tout aussi indispensable à sa mise en place.

Il y aurait donc toujours la notion d'interaction entre le sujet, ses compétences intrinsèques, son vécu passé traumatique, et puis les conditions de son présent, avec tout ce que comporte le monde extérieur d'agents différents (politiques, économiques, sociaux, humains) en interaction continue.

Il faut cependant admettre que la majorité des recherches réalisées dans le domaine de la résilience prennent en considération l'aspect dynamique du phénomène. La résilience étant une capacité à faire face, à se développer et à s'adapter en régénérant ses forces et ses compétences, à investir son propre potentiel et interagir avec les facteurs favorisants externes, ne pourrait être approché que d'une manière multidimensionnelle, tel un processus dynamique prenant en compte les aspects physiques, cognitifs, affectifs, sociaux et culturels.

Cette complexité dans l'approche de la résilience comme processus dynamique a été évoqué par Guedeney en 1998 : « la résilience est donc bien un processus complexe, un résultat, l'effet d'une interaction entre l'individu et son environnement. Et l'aspectclef de cette relation, c'est bien semble-t-il la capacité d'être en relation avec l'autre : on n'est pas résilient face à tout et n'importe quoi et on ne l'est en tout cas pas tout seul, sans être en relation ».

De plus, cet auteur met l'accent sur le fait que la résilience ne peut pas être statique, dans le sens où l'être humain ne devient pas 
résilient tout le temps et pour toujours, ni face à toutes adversités, et encore moins en comptant sur ses propres ressources.

C'est donc plutôt les recherches de type longitudinal ou rétrospectif, qui ont permis cette constatation. La résilience serait un processus acquis progressivement et soumis à des variabilités face à différentes circonstances rencontrées par l'individu. Cette approche dynamique de la résilience se réfère à la psychologie développementale, la psychologie de la santé et à la psychanalyse, celle-ci s'intéressant aux processus intrapsychiques impliqués dans la résilience.

L'étude longitudinale de Werner a pu mettre en évidence la dynamique exprimée dans le processus de résilience, n'étant pas stable ni définitive, elle serait le résultat d'un équilibre entre les facteurs, internes et externes, dits de risques et d'autres de protection en présence de conditions difficiles ou de traumatisme. (M. Anaut, 2008, p.45).

La résilience est de ce fait également un équilibre entre les différents facteurs individuels, familiaux et sociaux, en interaction, en plus d'être un processus évolutif adaptatif aux circonstances nouvelles qui pourraient avoir une incidence négative, relativement grave, sur le développement harmonieux de l'individu.

$\mathrm{Au}$ terme de ce que nous venons de relater, nous pouvons dire qu'il n'existe pas de définition unifiée de la résilience. Chacun de ces chercheurs aurait une version qui insisterait sur l'un ou l'autre deses aspects, en s'appuyant sur telle ou telle approche. 
Cependant le fait de se limiter à une définition de la résilience bien cadrée n'engage en rien l'exclusion des autres dimensions du concept, mais cela représente uniquement une obligation pour le chercheur de se positionner dans le champ de sa recherche, ceci pour mieux cerner les limites du phénomène étudié.

Nous pouvons retenir cette définition qui aurait ciblé certains points que d'autres : la présence de traumatisme, la reprise du développement positif, l'interaction avec l'environnement.

Pour plusieurs auteurs (Cyrulnik, De Tychey, Lighezzolo), le traumatisme est une condition primordiale à l'apparition de la résilience. Dans cet abord Cyrulnik écrit dans son ouvrage « le murmure des fantômes » qu' : «On ne peut parler de résilience que s'il y a eu un traumatisme suivi de la reprise d'un type de développement, une déchirure raccommodée ».(B. Cyrulnik, 2003, p.19).

Il est donc impératif que le sujet soit traumatisé par un évènement donné et qu'il ait pu reprendre son développement pour qu'on puisse parler de son éventuelle résilience. Dans ce cas ne sont pris en compte que les évènements assez bousculant, de manière à déstabiliser l'équilibre bio-organique ou psychique de l'individu, tel un accident grave causant des infirmités d'ordre physique ou mental, des catastrophes naturelles, des agressions physiques ou sexuelles, une perte soudaine et violente d'un être cher, etc.

En final nous pouvons définir la résilience, comme étant un processus dynamique à visée adaptative et évolutive, se déclenchant 
suite à un incident traumatique pouvant provoquer de graves conséquences dans la vie d'un individu, qui serait dès lors capable de reprendre son cours de développement dans un sens positif. Ceci en puisant dans ses ressources individuelles ainsi qu'à travers l'interaction de différents aspects environnementaux dans un but de réaliser un équilibre entre les facteurs intrinsèques et extrinsèques.

\section{b) Le Contexte descriptif du profil résilient :}

Afin de décrire le processus de résilience, Rutter distingue trois caractéristiques principales chez les sujets résilients en présence de conditions psychosociales défavorables.

Ces caractéristiques témoignent de la mise en route d'un fonctionnement spécifique qui donne lieu à certains comportements déterminés.La première caractéristique serait l'estime de soi.Lorsqu'elle est satisfaisante, celle-ci signerait la bonne adaptation sociale, elle serait nécessaire à l'affrontement du stress occasionné par l'adversité. Pouvoir se définir pour un individu, et avoir le sentiment de sa propre valeur contribuent à la construction d'une bonne estime de soi, et ainsi à développer un processus résilient en cas de traumatisme.

Rogers et ses collaborateurs définissent l'estime de soi comme le « moteur de l'activité sociale, en tant que source de satisfaction, de sécurité consciente ou inconsciente à connotation affective, positive ou négative, étroitement liée à des processus cognitifs ».(A.Lamia et S. Esparbès-Pistre, 2004, p. 92). 
Cette définition démontre un jugement d'ordre affectif porté sur soi, négatif ou positif, il renvoie à la perception d'une satisfaction et d'un sentiment de sécurité consciente ou inconsciente et qui serait lié aux processus cognitifs.

Cette évaluation de la perception de soi a été évoquée sur un plan dynamique par Coopersmith, « elle constitue l'expression d'une approbation ou d'une désapprobation portée sur soi-même » (A.Lamia et S. Esparbès-Pistre, 2004).

Un individu qui aurait une bonne estime de soi, se verrait comme quelqu'un d'important, qui aurait des capacités et des compétences et qui serait accepté dans la société. Ses comportements et ses opinions se réfèrent à son auto-estime dans son rapport avec le monde.

A l'inverse une mauvaise estime de soi, aurait un impact négatif sur l'intégration sociale de l'individu, et entraverait son adaptation, par le fait de diminuer ses potentialités, limiter ses actions et freiner l'acquisition de compétences.

La deuxième caractéristique concerne le sentiment d'autoefficacité. Cette qualité de pouvoir anticiper et d'être conscient de son efficacité à résoudre les problèmes, fait partie des capacités qui donne à l'individu confiance en lui et en ses compétences lui permettant de se projeter dans l'avenir et aller de l'avant. Ceci s'inscrit justement dans la trajectoire de la résilience. 
Selon Rutter les différentes expériences d'ordre socioéducatif contribueraient à renforcer ou à réprimer ce sentiment. Le rôle des parents étant en premier lieu la source des premières relations qui influencent grandement le développement chez l'enfant.

Celui acquiert au fur et mesure le contrôle interne ainsi prendt-il conscience de ses propres compétences à réaliser ses projets. Le développement de l'autonomie de l'enfant dépendra essentiellement, au cours de son jeune âge, du type de «parenting »1(Parentalité). Suivant les méthodes éducatives employées avec l'enfant, il sera capable de faire preuve d'indépendance et d'efficacité, ou inversement, il ne saura pas accomplir des taches sans assistance de la part d'un tiers.

Le relai du parenting, dans la construction de l'auto-efficacité et la résolution des problèmes chez l'enfant, sera pris par les partenaires sociaux qui s'impliqueront dans l'accompagnement socioéducatif de l'enfant moins jeune, en développement continu, et en ouverture sur le monde extérieur.

La troisième caractéristique étant la capacité de l'individu à résoudre les problèmes sociaux. Cela reviendrait à sa capacité de tenir compte de ses expériences passées, et d'en tirer des stratagèmes pour pouvoir se comporter positivement face à des contraintes sociales.

1Parenting: Fonction de parent, notamment sur les plans juridique, moral et socioculturel. (Larousse, 2008, p. 743). Terme utilisé par les psychanalystes et développementalistes désignant le processus qui mène à l'état d'être parent. C'est Racamier qui introduit, en 1961 pour la première fois le terme de parentalité et maternalité dans un article qui tentait d'expliquer les causes de la psychose puerpérale. (C. Cassagne, 2006) 
Ces expériences peuvent être d'ordre personnel, familial ou extrafamilial, le sujet donc puise son ressourcement face à l'adversité, non seulement à partir de ses compétences internes, mais également depuis son milieu direct (famille, entourage proche) ou indirect (communauté sociale). Un sujet résilient serait tout à fait capable de la résolution des problèmes sociaux qui le rencontrerait et d'en dépasser les entraves à son adaptation, en s'appuyant sur l'appartenance à un contexte socio- relationnel riche en ressources positives.

Gilligan a proposé un autre modèle de la construction de la résilience issu de ses travaux qui regroupent plus d'une discipline (psychologie du développement, psychologie sociale). Ce modèle n'est pas très différent de celui de Rutter, il y a rajouté, aux domaines de l'estime de soi et de l'auto-efficacité faisant partie du modèle cité plus haut, le domaine de la base de sécurité qui renvoie à la théorie de l'attachement.

Avoir le sentiment de sécurité interne, est pour ce chercheur, lié aux premières expériences de la vie, ainsi aux premiers liens d'attachement. Cette sécurité interne participerait au développement de la résilience par le fait de se sentir soutenu et reconnu. Certains chercheurs considèrent qu'un individu carencé sur le plan familial pourra pallier à cette défaillance dans l'établissement de liens d'attachement par un bon étayage social (milieu extrafamilial, scolaire, professionnel, etc.), ceci pouvant renforcer la base de sécurité. (M. Anaut, 2008, p.73). 


\section{La résilience, un équilibre entre les facteurs de risque et les facteurs de protection :}

Comme nous avons pu en débattre plus haut, la résilience ne peut avoir une seule définition. Il y a plusieurs approches de la résilience, et chacune soutien un des aspects, vu sous un angle épistémologiquement différent, selon la nature de l'étude, et l'intérêt portée à celle-ci. Nous avons également pu discuter le fait que la résilience ne peut être définitive, c'est-à-dire acquise tout le temps et devant n'importe quelle situation traumatisante.

Les individus pouvant être résilients dans une période de leur vie et pas dans une autre, cela dépendrait non seulement de l'état psychologique, développemental, cognitif, affectif du sujet, mais également des influences externes émanant de son environnement et qui influenceraient ses ressources. Ces facteurs internes et externes présents, chez le sujet ou dans son environnement, et qui peuvent être de risques ou de protection, auraient un rôle à jouer dans l'équilibre issu de l'interaction dynamique de ces facteurs qui construirait la résilience.

On a pu, grâce à des études longitudinales comme celle d'E. Werner, démontrer que malgré les conditions défavorables dans lesquelles vivaient les enfants, il y avait entre eux ceux qui réussissaient. Nous pouvons également citer les études effectuées dans le domaine socioéducatif pour la prise en charge précoce des enfants appelés de "mséf »; c’est les enfants qui viennent de milieux socioéducatifs faibles, (ex : le programme américain : Head Start, le 
programme québécois : l'Opération Renouveau), celles-ci prennent en considération l'évolution de l'enfant dans son environnement social, ceci implique la prise en compte de sa famille, de son entourage, de sa vie sociale et de son milieu scolaire. Ces recherches sont arrivées à démontrer qu'un certain nombre d'enfants de mséf, bien que confrontés à des conditions environnementales très défavorables sur le plan socioéconomique, étaient socialement bien adaptés et performants sur le plan scolaire, ceci leur a valu un statut d'enfants «atypiques» dans leur milieu. C'est ce qui a suscité l'intérêt à identifier les facteurs qui auraient permis ce changement positif dans la trajectoire du développement psychosocial de ces enfants dits «vulnérables ». (Terisse et Larose, 2001, P. 3).

La présence donc de facteurs de protections capables d'interagir avec les facteurs de risques présents chez le sujet lui-même ou dans son environnement, et de lui permettre de s'adapter à ses conditions défavorables, intéresse davantage les études menées dans le domaine de la résilience et dans la compréhension de la mise en route de ce processus chez les individus.

Dans cette perspective, Tap et Vinay ont écrit : «Dans la résilience, l'individu développe des mécanismes de protection qui réduisent l'incidence du risque en exerçant des effets sur le risque luimême ou en modifiant le fait d'être exposé ou de s'exposer au risque. Ces mécanismes réduisent la probabilité qu'une réaction en chaîne négative soit déclenchée par la rencontre avec le risque. Ils renforcent, par ailleurs, l'estime de soi et l'efficacité personnelle par 
l'intermédiaire de solides relations personnelles de soutien ou grâce à la réussite dans l'accomplissement de tâches». (Terisse et Larose, 2001, p. 13).

D'après cela, nous pouvons dire que la résilience serait un processus où l'individu face à l'adversité, développe des mécanismes de protection pour essayer de contrer les risques d'inadaptation, accourus ou d'en réduire l'effet négatif d'un côté, et de promouvoir les ressources personnelles et environnementales (estime de soi, compétences sociales, soutien social) d'un autre côté.

Il apparait indispensable donc d'essayer de comprendre ces deux concepts directs et indirects, facteurs de risques et facteurs de protection, susceptibles d'évoluer dans le temps selon le contexte, ainsi que leur interaction dynamique relative à l'acquisition de l'équilibre adaptatif de la résilience. Selon cette approche, la personne serait résiliente s'il y aurait un équilibre issu de l'interaction entre les facteurs individuels et les facteurs environnementaux. Mais quels seraient donc ces facteurs de risques et facteurs de protections impliqués dans cette dynamique complexe de la résilience?

\section{a) Facteurs de risques et vulnérabilité :}

Le risque implique la présence d'une caractéristique liée à la personne elle-même, à son environnement, sa culture, son mode de vie, entraînant une probabilité plus élevée de développer une maladie ou à subir un dommage ou une perte, que cela soit sur le plan physique, psychologique ou socioéconomique. 
L'organisation mondiale de la santé définit le facteur de risque comme étant « tout attribut, caractéristique ou exposition d'un sujet qui augmente la probabilité de développer une maladie ou de souffrir d'un traumatisme ». (http//:Oms.fr, 2012, 10h:34).

En psychologie, les facteurs de risques seraient incriminés dans l'inadaptation des sujets à leur environnement ainsi qu'à l'apparition d'affections psychopathologiques ultérieures.

Selon Garmezy, cité par Terisse et Larose dans un article publié dans le Cahiers du Centre de recherche sur les formes d'éducation et d'enseignement, les facteurs de risque sont définit comme « les facteurs dont la présence accroît la possibilité qu'un enfant ait des difficultés d'adaptation et d'apprentissage si on le compare à un enfant de la population générale ». Ceci impliquerait que l'évolution d'un sujet dans un milieu comportant des risques entraverait son adaptation et son développement « normal » ultérieur.

Cité par Anaut (2003), ce même auteur définit les facteurs de risque comme étant« un évènement ou une condition organique ou environnementale qui augmente la probabilité pour l'enfant de développer des problèmes émotifs ou de comportements ». Ces facteurs de risques pourraient être d'une nature organique, se référant à l'individu lui-même et à ses qualités personnelles, ou provenant de son environnement psychosocial, économique et culturel, et qui tendent à développer chez l'enfant des troubles d'ordre affectif ou comportemental. 
D'après l'étude de Werner et Smith, un enfant qui cumulerait plus de quatre facteurs de risque à l'âge de deux ans, aurait plus de difficultés à s'adapter plus tard (dix ans) que les autres enfants.

Ce cumul de facteurs de risque est selon Terisse et Larose, serait la cause d'apparition de stress élevé chez l'individu. Ils se réfèrent à Lazarus et Folkman pour définir le stress comme: « un état de tension résultant d'interactions négatives entre l'enfant et son environnement. Il est confronté à des situations adverses que ses ressources personnelles ne lui permettent pas de contrôler et qui deviennent, de ce fait, menaçantes, d'où la probabilité que, dans sa recherche d'équilibre avec son environnement, il adopte des stratégies d'adaptation («coping») inadéquates se traduisant, par exemple, par des difficultés comportementales. Dans le cas contraire, si la mobilisation de ses ressources personnelles et de celles de son environnement lui permet de développer des stratégies adéquates et couronnées de succès, nous parlerons de résilience ».(Terisse et Larose, 2001, p. 9).

Cette définition montre donc clairement que la résilience représente l'ensemble de réponses positives (adéquates et couronnées de succès) aux stress engendré par le cumul de facteurs de risque qui devient menaçant dès lors qu'il entrave sa recherche d'équilibre avec son environnement. Les facteurs de risque ont dans ce cas, un effet cumulatif et interactif, un sujet étant exposé à plusieurs facteurs de risque sera considéré comme sujet à risque élevé, vulnérable et aurait tendance à adopter une trajectoire de vie négative. 
Un facteur de risque isolé aurait alors un effet moindre sur la vulnérabilité de l'individu, que s'il interagissait avec d'autres facteurs de risque. Car l'effet additionnel de plusieurs facteurs de risque rend plus menaçante la situation défavorable dans laquelle vit le sujet. De plus l'interaction entre les facteurs de risques eux-mêmes, multiplie l'effet de l'un et de l'autre en leurs procurant un impact plus néfaste (ex : l'addiction peut mener aux agressions sexuelles, et cette dernière aurait une incidence sur l'augmentation du recours à la première).

La mise en évidence des facteurs de risque a suscité l'intérêt de plusieurs chercheurs, il y en a même d'entre eux qui ont tenté de dresser un profil de risque (Athony) donnant naissance à une pathologie, ou encore de construire des échelles (Samerof) pour évaluer les facteurs de risque, en qui la présence, empêcherait l'individu à s'adapter. Cependant il y eu des controverses à cette idée de l'incapacité des personnes à s'adapter en présence de certains facteurs de risque et même des plus important. Garmezy a renié le fait qu'un développement adapté serait impossible en présence de conditions de risque, car cela remettrait en question la capacité de résilience chez certains individus.

La vulnérabilité des personnes mise en évidence à travers la présence de facteurs de risque ne remet donc pas en question leur possibilité de devenir « résilients ». Le sujet étant capable de puiser dans ses ressources personnelles et celles de son environnement, ceci est l'expression de son mode de défense ; facteurs de protection, sans lesquels nous ne pourrions envisager une réponse en présence de 
conditions menaçantes. Cette dernière serait la résultante d'interaction entre les facteurs de risque et les facteurs de protection.

Les facteurs de risque ne peuvent donc pas agir seuls pour déterminer le devenir de l'être humain. C'est pour cela que la prise en compte des facteurs de protection est tout aussi indispensable dans la perspective de résilience des individus en détresse.

\section{b) Les facteurs de protection et La résilience :}

Selon Rutter, les facteurs de protection seraient des variables qui modifieraient les réponses des individus aux situations menaçantes en réduisant les effets du risque et les réactions négatives. Ceci implique qu'en présence d'adversité le sujet réagirait différemment au risque présent si un facteur de protection s'y interpose. Ce dernier aurait un rôle de modérateur du risque, ainsi il réduirait sa nocivité et aiderait à mieux faire face à la situation stressante.

Les facteurs de protection seraient des caractéristiques personnelles (génétique, biologique ou psychologique), ou des conditions environnementales (implication des parents, soutien social) qui agiraient sur l'incidence négative des facteurs de risque pour en diminuer l'effet dans le but d'arriver à un équilibre adaptatif.

Le fait de contrer l'effet du risque ne fait pas des facteurs de protection des opposés aux facteurs de risque. Les facteurs de protection ont un rôle d'atténuation de la nocivité en procurant un support à l'individu pour pouvoir se développer et évoluer sainement malgré les conditions difficiles. Si un sujet évoluerait dans un milieu 
défavorable, la présence de protection dans l'un des aspects de son environnement pourrait l'aider à résister aux contraintes et adversités subies par les autres aspects de son milieu.

Parmi les études réalisées dans le domaine des facteurs de protection présents chez l'être humain et son entourage, nous citons celle de Garmezy et Masten en 1991, qui sont arrivés, à partir d'observations de familles défavorisées, à identifier trois domaines de protection sur lesquels reposerait la résilience :(M. Anaut, 2008, p-p 40-41).

$>$ Les facteurs de protection individuels :

Tempérament actif, doux, gentil, genre (être une fille avant l'adolescence ou un garçon durant l'adolescence.), âge (jeunesse), QI élevé, ou bon niveau d'aptitudes cognitives, Sentiment d'autoefficacité et d'estime de soi, compétences sociales, conscience des relations interpersonnelles (proche de l'intelligence sociale), sentiment d'empathie, locus de contrôle interne, humour, attrayant pour les autres.

$>$ Les facteurs de protection familiaux :

Parents chaleureux et soutien parental (au moins un des parents), bonnes relations parents/enfants., harmonie parentale (entente)

$>$ Les facteurs de protection extrafamiliaux :

Réseau soutien social (grands-parents, pairs), Expériences de succès scolaire. 
Ils font également remarquer que certains de ces facteurs de protection sont internes et concerneraient les ressources personnelles de l'individu, et d'autres seraient issu de l'interaction avec l'environnement. Aussi font-ils préciser (Garmezy et Masten) que les mêmes facteurs de protection ne sont pas présents chez chaque individu, ils seraient variables selon l'âge et la période du développement, et les sujets n'en font pas le même usage.

A cet effet il ne faut pas se limiter à l'énumération de facteurs de protection car ceci mettrait de côté l'aspect contextuel dans lequel ils opèrent. Cette observation concernerait tout concept évoluant dans une dynamique interactionniste. C'est le cas type du processus de la résilience qui renferme une dimension adaptative et évolutive.

Facteurs de risque et facteurs de protection n'étant pas contraires, ils peuvent même parfois concerner un seul aspect, à la différence du degré de maturité de l'aspect lui-même, de la période du développement de la personne ou de la fonctionnalité chez les individus.

D'une manière générale les facteurs de protection se décriraient comme des caractéristiques, des habiletés, des capacités, des conduites, d'origine individuelle, familiale ou extrafamiliale, qui protègeraient la personne contre les effets négatifs d'une situation traumatisante, stressante pour en réduire l'impact et en favoriser l'adaptation en s'inscrivant dans une perspective de résilience. Il faut juste souligner que les facteurs de risque ne se limiteraient pas aux aspects individuels comportementaux car il ne faut pas oublier le rôle 
des aspects intrapsychiques de la personne et qui ont leur rôle de la protection de son intégrité (les mécanismes de défenses).

En final, si nous nous en tenons à la perspective que la résilience serait la résultante de l'équilibre dynamique établi entre les facteurs de risque et les facteurs de protection. Nous soutenons l'idée que l'individu ne nait pas résilient et ne le sera pas à l'infini ni contre tout. Cela dépondra du contexte présent relatif à la situation en question. Il est indispensable donc qu'un individu exposé à un certain nombre de risque, développe des aptitudes, des défenses pour se protéger de l'adversité, et éviter l'apparition de conduites ou d'attitudes pathologiques. 


\section{- Bibliographie :}

1. ANAUT Marie (2003), « La résilience : surmonter les traumatismes », Paris,Nathan/VUEF.

2. ANAUT Marie (2006), « La résilience au risque de la psychanalyse, ou la psychanalyse au risque de la résilience ? », in B. Cyrulnik et Ph. Duval, Psychanalyse et résilience, Paris, Odile Jacob.

3. ANAUT Marie (2008), « La résilience : surmonter les traumatismes », Paris, $2^{\text {ème }}$ éd., Armand Colin.

4. BAILLY Lionel (2006), « Résilience et Psychanalyse », in B. Cyrulnik et Ph. Duval, Psychanalyse et Résilience, Paris, Odile Jacob.

5. CASSAGNE C.(2006), « La parentalité dans les lieux d'accueil de la petite enfance », Ed. Weka.

6. CYRULNIKBoris(1999), « Un Merveilleux Malheur »,Paris, Odile Jacob.

7. CYRULNIKBoris (2003), « Le Murmure Des Fantômes », Paris, Odile Jacob.

8. CYRULNIK Boris (2011), in Serban IONESCO, «préface dans Traité de résilience assistée », 1 ère éd. Paris, Quadrige/PUF.

9. LAMIA A.et ESPARBES-PISTRE S.(2004), «Estime De Soi Et Vulnérabilité, Chapitre 6 », in P. TAP et Maria De Lourdes Vasconcelos, Précarité et vulnérabilité psychologique, Paris,ERES.

10. LEMAYMichel (2001), « La Résilience devant la Violence », article publié dans la Revue Québécoise de Psychologie, Vol. 22, n 1 .

11. SILLAMYNorbert (2006), « Dictionnaire de psychologie », éditionLarousse. 
12. TERISSE B.et LAROSEF. (2001), «La résilience : facteurs de risques et facteurs de protection dans l'environnement social et scolaire du jeune enfant »,article publié par le Centre de Recherche sur les Formes d'Education et d'Enseignement, numéro thématique, École/Famille : Quelle médiation? XIV, 129-172.

13. THEISAmandine (2006), «Approche psychodynamique de la Résilience ", Thèse de Doctorat en psychologie clinique, dirigée par Pr. Claude de Tychey, université de Nancy 2.

14. TOMKIEWICZStanislaw (2002), «L'émergence du concept », in B. Cyrulnik et coll., La résilience : le réalisme de l'espérance, Paris, Erès.

15. Le petit Larousse illustré (2008), Paris,édition-Larousse.

16. Facteurs de risques, http//:Oms.fr, 21/12/2012, 10h:34. 